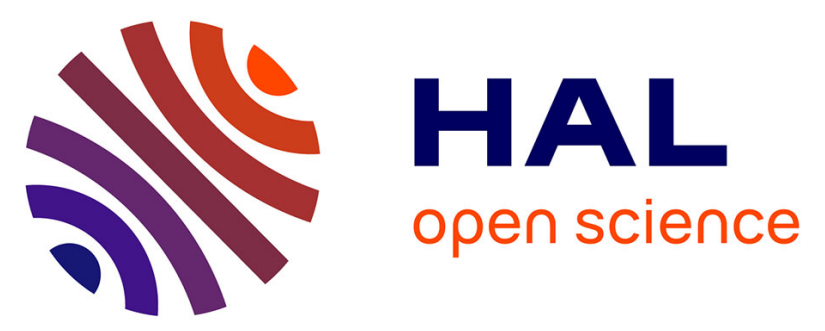

\title{
How Electrostatics Influences Hydrodynamic Boundary Conditions: Poiseuille and Electro-osmostic Flows in Clay Nanopores.
}

Alexandru Boţan, Virginie Marry, Benjamin Rotenberg, Pierre Turq, Benoit Noetinger

\section{To cite this version:}

Alexandru Boţan, Virginie Marry, Benjamin Rotenberg, Pierre Turq, Benoit Noetinger. How Electrostatics Influences Hydrodynamic Boundary Conditions: Poiseuille and Electro-osmostic Flows in Clay Nanopores.. Journal of Physical Chemistry C, 2012, 117 (2), pp 978-985. 10.1021/jp3092336 . hal-01484359

\section{HAL Id: hal-01484359 \\ https://hal.sorbonne-universite.fr/hal-01484359}

Submitted on 13 Nov 2018

HAL is a multi-disciplinary open access archive for the deposit and dissemination of scientific research documents, whether they are published or not. The documents may come from teaching and research institutions in France or abroad, or from public or private research centers.
L'archive ouverte pluridisciplinaire HAL, est destinée au dépôt et à la diffusion de documents scientifiques de niveau recherche, publiés ou non, émanant des établissements d'enseignement et de recherche français ou étrangers, des laboratoires publics ou privés. 


\title{
How Electrostatics Influences Hydrodynamic
} Boundary Conditions: Poiseuille and Electro-Osmostic Flows in Clay Nanopores.

\author{
A. Boţan, ${ }^{*}, \dagger$ V. Marry, ${ }^{\dagger}$ B. Rotenberg,${ }^{\dagger}$ P. Turq,${ }^{\dagger}$ and B. Noetinger ${ }^{\dagger}$ \\ UPMC Univ Paris 06, CNRS, ESPCI, UMR 7195 PECSA, F-75005 Paris, France \\ , and IFP - Energies nouvelles, 1-4 Avenue de Bois-Préau, Rueil-Malmaison 92852, France \\ E-mail: alexandru.botan@gmail.com
}

${ }^{*}$ To whom correspondence should be addressed

UPMC and CNRS

†IFP-EN 


\begin{abstract}
We report on a molecular simulation study of the origin of non-slip or slip hydrodynamic boundary conditions in clay nanopores, focussing on the role of electrostatics. We simulate hydrodynamic and electro-osmotic flows and consider both charged (montmorillonite) and uncharged (pyrophyllite) clays. We further use two commonly used force fields to analyze the effect of local interactions, in particular the effect of the polarity of the surface, in addition to the mere effect of the presence or absence of a net charge and counter-ions. For the $6 \mathrm{~nm}$ pore investigated here, the molecular velocity profile can be well described by continuum hydrodynamics only if (a) proper boundary conditions, with a slip or stagnation length determined from molecular simulation, are taken into account and (b) the ionic density profiles from MD simulations are used in the case of electro-osmotic flow, since the Poisson-Boltzmann equation fails to reproduce the ionic profiles, hence the force acting on the fluid. Among the considered force fields only CLAYFF predicts a hydrophobic pyrophyllite and hydrophilic montmorillonite, as expected from experimental behaviour. The non-slip or slip boundary conditions at clay surfaces strongly depend on electrostatic interactions of water molecules with the surface. The presence of a net charge results in an average electric field experienced by surface water molecules between the charged surface and the condensed layer counter-ions, which influences their orientation. The charge distribution inside the clay layer determines the polarity of the surface and hence the strength of hydrogen bonds donated by water molecules to surface oxygen atoms.
\end{abstract}

Keywords: molecular simulation, water/solid interface, charged surface, Poisson-Boltzmann theory, dynamics 


\section{Introduction}

The transport properties of fluid in clay minerals are of great practical importance. On the one hand, the low permeability of clays makes them attractive barriers for the storage of carbon dioxide or toxic chemical and radioactive waste. On the other hand, the slow fluid migration hinders the recovery of shale gas and oil. The theoretical description of fluid transport in clays is difficult because of the multiscale structure of these porous material with a wide range of pore sizes, usually classified as interlayer $(<2 \mathrm{~nm})$, interparticle $(2-50 \mathrm{~nm})$ and intergranular $(>50 \mathrm{~nm})$ pores. Moreover, adsorption/desorption processes between clay layers can lead to the swelling or shrinkage of clay particles and thereby affect the permeability of the material. Multiscale approaches, both from the theoretical and experimental points of view, are required to understand the transport of fluids through clays.

Quasi-elastic neutron scattering ${ }^{1-3}$ and molecular dynamics simulations ${ }^{4-6}$ have shown that the transport of water confined in interlayer pores is purely diffusive. This motion between clay layers strongly depends on many factors such as the clay type, the nature of the counter-ions, the presence of other molecules, temperature, etc. ${ }^{7-10}$ Transport on the macroscopic scale is usually described by Darcy's law, arising from convective flow in the larger pores coupled to the complex geometry of the pore network; the influence of microscopic details such as the chemical composition is less pronounced.

For interparticle pores of a few nanometers, convective flows are more sensitive to the details of the water/clay interactions. The latter strongly depend on the nature of the clay, in particular on whether the mineral layer is charged or not. From a static point of view, most uncharged clays behave as hydrophobic (e.g. large contact angles) while with charged clays the presence of counterions induces hydrophilic behaviours (e.g. swelling in the presence of water). Studies focussing on the dynamic aspects of this interaction (non-slip or slip) in clays are scarce and one thus usually assumes a correspondence between the hydrophilic/phobic static properties and non-slip/slip in the presence of a flow. Contact angle measurements may depend on the experimental technique and other factors such as surface roughness, fluid viscosity, electrical properties, gas bubbles, pressure, 
etc. ${ }^{11,12}$ Nevertheless, Huang et al. found a quasi-universal relationship between water slippage and the contact angle on model hydrophobic surfaces. ${ }^{13}$

The hydrodynamic boundary conditions are determined by a number of factors, including electrostatic ones. Cho et al. have shown experimentally that the slip length is primarily influenced by the dipole moment of the fluid rather than the wettability of the liquid at the surfaces, and reported a decrease in the slip length with increasing dipole moment. ${ }^{14}$ As an explanation, the authors suggested that the dipole-dipole interactions result in a "surface lattice structure" in the liquid near the surface. Stein et al. studied the surface charge dependence on the ionic transport in nanochannels via electro-osmotic flow and found that the ionic liquid conductivity increases with increasing surface charge of the channel. ${ }^{15}$ Dukhin et al. measured $\zeta$-potentials for alumina-KCl interfaces and showed that an electric double layer can even be observed at uncharged surfaces. ${ }^{16}$ Joseph and Aluru went into details of this effect using molecular simulations. ${ }^{17}$ For this purpose, two neutral silica slit channels with and without partial charges filled by $1 \mathrm{M} \mathrm{KCl}$ were modeled. The presence of an electrical double layer and electro-osmotic flow were observed for both channels. The presence of the ions and the wall partial charges change the interfacial orientation of water from that of water near an uncharged surface affecting the properties of the electric double layer near the interface.

Clays provide an interesting class of systems to investigate the microscopic origin of the nonslip/slip behaviour at a mineral surface. Indeed, they cover ranges of nearly identical systems which may vary by the presence or absence of charge, by the charge distribution inside the solid and by the nature of the counter-ions. The non-slip or slip nature of hydrodynamic boundary conditions in montmorillonite clay nanopores has been studied by molecular simulations for both Poiseuille and electro-osmotic flows. In order to investigate the non-slip/slip behaviour at such surfaces, Marry et al. ${ }^{18}$ and Dufrêche et al. ${ }^{19}$ have used equilibrium molecular dynamics simulations and Kubo relations to compute electro-osmotic flows. The main conclusion was that the Stokes equation correctly predicts these flows only if slip boundary conditions are taken into account. In a recent study of hydrodynamic (Poiseuille) water flow through Na-montmorillonite nanopores, Botgan et al. 


\section{Systems and methods}

\section{Microscopic model}

Two different types of clay are considered: pyrophyllite, a dioctahedral 2:1 neutral clay with unit formula $\mathrm{Al}_{4} \mathrm{Si}_{8} \mathrm{O}_{20}(\mathrm{OH})_{4}$, and montmorillonite, a cationic clay, which can be obtained from pyrophyllite by partial isomorphous substitution of $\mathrm{Al}^{3+}$ ions by $\mathrm{Mg}^{2+}$ ions in the octahedral layers to give a layer composition of $\mathrm{Na}_{0.75}\left[\mathrm{Si}_{8}\right]\left(\mathrm{Al}_{3.25} \mathrm{Mg}_{0.75}\right) \mathrm{O}_{20}(\mathrm{OH})_{4}$. Counterions $\mathrm{Na}^{+}$in the interlayer region compensate the negative charge of clay layers. The simulation box contains two clay layers, forming a $4.5 \mathrm{~nm}$ slit pore centered at $z=0$, water molecules distributed in the pore and $\mathrm{Na}^{+}$ions, the latter only for montmorillonite. Each clay layer contains $8 \times 4 \times 1$ unit cells (totalling 1280 atoms) with dimensions $41.44 \times 35.88 \times 6.54 \AA^{3}$. The number of water molecules was chosen as a result of a prior grand canonical Monte Carlo simulation as presented in our previous study: ${ }^{20}$ 
2437 (resp. 2474) for montmorillonite with CLAYFF (resp. SSFF) and 2437 (resp. 2478) for pyrophyllite with CLAYFF (resp. SSFF). Figure 1 illustrates the simulation box. Periodic boundary conditions are applied only in the $x$ and $y$ directions and empty space was added in the $z$ direction.

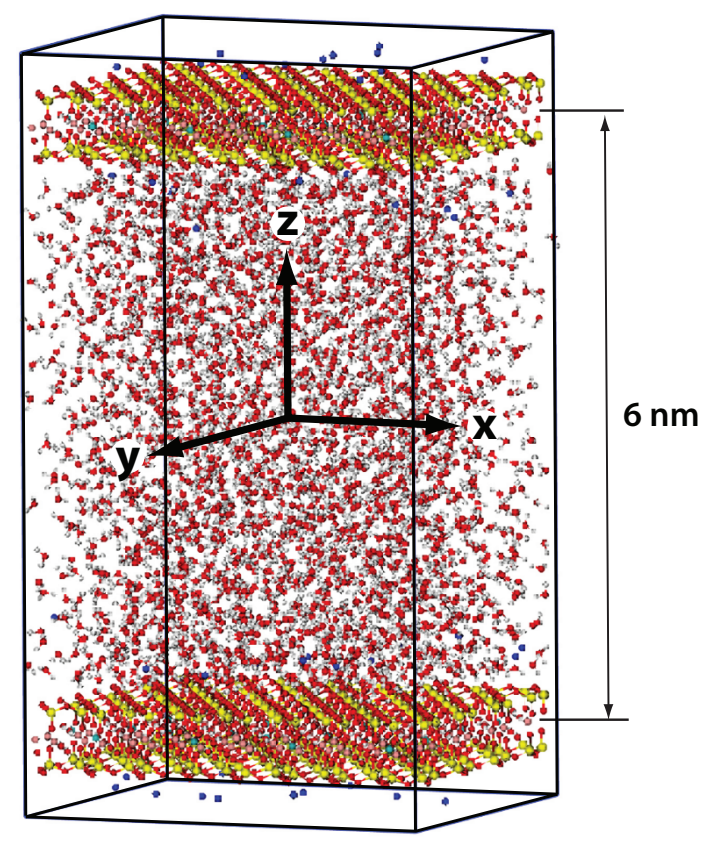

Figure 1: Snapshot of the simulation box for montmorillonite: pink, Al; green Mg; yellow, Si; red, O; white, H; blue, Na.

Several force fields have been proposed to describe clay materials. ${ }^{21-29}$ They generally (but not always) describe the interactions between atoms by pairwise additive Lennard-Jones and Coulomb potentials:

$$
V_{i j}=\frac{q_{i} q_{j}}{4 \pi \varepsilon_{0} r_{i j}}+4 \varepsilon_{i j}\left[\left(\frac{\sigma_{i j}}{r_{i j}}\right)^{12}-\left(\frac{\sigma_{i j}}{r_{i j}}\right)^{6}\right]
$$

where $r_{i j}$ is the distance between sites $i$ and $j$ of different molecules, $q_{i}$ is the partial charge of the site, and $\sigma_{i j}$ and $\varepsilon_{i j}$ are LJ parameters deduced from the conventional Lorentz-Berthelot mixing rules. ${ }^{30}$ In the present work, we consider two of the most commonly used force field to simulate clays, which are of the form described above but differ by the value of the corresponding parameters: (a) the one proposed by Skipper ${ }^{31}$ and subsequently adapted to the SPC/E water model by Smith, ${ }^{25}$ denoted as SSFF in the following, and (b) CLAYFF, introduced by Cygan et al. ${ }^{27}$ The LJ 
parameters and charges are presented in Table 1. Both force fields predict thermodynamic properties of clays in fair agreement with experiments, ${ }^{27,32-35}$ for diffusion coefficients better predictions are obtained with CLAYFF. 9,36

In order to study the influence of electrostatics on boundary conditions the clay substrate, we treated the clay as rigid for both force fields. While CLAYFF allows to describe the flexibility of the clay framework, this is not possible with SSFF. For a discussion of the effect of flexibility on boundary conditions, using the CLAYFF force field, we refer the reader to Ref. 37. Water molecules are described using the SPC/E (Simple Point Charge/Extended) model, ${ }^{38}$ while $\mathrm{Na}^{+}$ cations are modeled using the parameters proposed by Smith and Dang. ${ }^{39}$

Table 1: Charges $q$ and Lennard-Jones parameters $\sigma$ and $\varepsilon$ of montmorillonite and pyrophyllite for SSFF and CLAYFF

\begin{tabular}{ccccccc}
\hline & \multicolumn{4}{c}{ SSFF } & \multicolumn{3}{c}{ CLAYFF } \\
specie & $q_{i}(\mathrm{e})$ & $\sigma_{i}(\AA)$ & $\varepsilon_{i}(\mathrm{kcal} / \mathrm{mol})$ & $q_{i}(\mathrm{e})$ & $\sigma_{i}(\AA)$ & $\varepsilon_{i}(\mathrm{kcal} / \mathrm{mol})$ \\
\hline octahedral aluminum & 3.0 & 0.0 & 0.0 & 1.5775 & 4.2712 & $1.3310^{-6}$ \\
octahedral magnesium (substituting Al) & 2.0 & 0.0 & 0.0 & 1.3598 & 5.26432 & $0.910^{-6}$ \\
tetrahedral silicon & 1.2 & 1.84 & 3.153 & 2.1 & 3.30203 & $1.8410^{-6}$ \\
hydroxyl oxygen & -1.0 & 3.166 & 0.156 & -0.95 & 3.16554 & 0.1554 \\
octahedral oxygen & -1.424 & 3.166 & 0.156 & & & \\
tetrahedral oxygen & -0.8 & 3.166 & 0.156 & & & \\
$\quad$ bridging oxygen & & & & -1.05 & 3.16554 & 0.1554 \\
bridging oxygen with octahedral substitution & & & & -1.1808 & 3.16554 & 0.1554 \\
hydroxyl hydrogen & 0.424 & 0.0 & 0.0 & 0.425 & 0.0 & 0.0 \\
\hline
\end{tabular}

\section{Non equilibrium molecular dynamics simulations}

In non equilibrium molecular dynamics, the force acting on a particle results from interparticle interactions and from an external force, e.g. from a gravitational or electrical field. In our simulations, we apply either (a) a constant force $F=0.75 \mathrm{cal} / \mathrm{mol} / \AA$, or (b) an electric field $E=0.01$ $\mathrm{V} / \AA$ in the $x$ direction (parallel to the clay layers) to simulate a steady hydrodynamic or electroosmotic flow, respectively. We verified the linear response of the system for all reported results. All simulations were carried out with the LAMMPS molecular dynamics package ${ }^{40}$ in the $N V T$ 
ensemble. The temperature was fixed at $300 \mathrm{~K}$ using a Nose-Hoover thermostat, coupled only to the degrees of freedom of the fluid in the $y$-direction, along the surface and perpendicular to the fluid flow. The velocities for $\mathrm{H}_{2} \mathrm{O}$ and $\mathrm{Na}^{+}$are collected every 0.1 ps for $6 \mathrm{~ns}$, in bins along $z$ of width $0.1 \AA$, after an equilibration period of $2 \mathrm{~ns}$.

\section{Continuous theory}

The traditional description of hydrodynamic flows and electrokinetic phenomena relies on a continuous description of the system, whereby the solvent is a continuum of viscosity $\eta$ and dielectric constant $\varepsilon_{r}$ and the charged surfaces are infinite walls with a uniform charge density. At steady state, the balance between viscous stresses and other forces is described by Stokes equation $\eta \Delta \mathbf{v}+\mathbf{F}_{\mathbf{v}}=\mathbf{0}$, where $\mathbf{v}$ is the velocity and $\mathbf{F}_{\mathbf{v}}$ the volumic force acting on the fluid. In the case of a hydrodynamic (Poiseuille) flow, the external force is a uniform pressure gradient along $x$. The profile $\mathbf{v}=v(z) \mathbf{e}_{\mathbf{x}}$ for the present case of a slit pore is parabolic. Defining $z=0$ as the $z$ position of the center of the pore and $\pm L_{\text {hyd }} / 2$ the position of the hydrodynamic interfaces, $v(z)$ reads:

$$
v(z)=v\left(L_{h y d} / 2\right)-\frac{F_{v}}{2 \eta}\left[z^{2}-\left(\frac{L_{h y d}}{2}\right)^{2}\right]
$$

The flow profile is then known when $v\left(L_{\text {hyd }} / 2\right)$ is set by the corresponding boundary condition, as will be discussed below.

In the case of electro-osmosis, $\mathbf{F}_{\mathbf{v}}$ arises from an applied electric field $\mathbf{E}=E \mathbf{e}_{\mathbf{x}}$, i.e. $\quad \mathbf{F}_{\mathbf{v}}=$ $c_{\text {ion }}(z) e \mathbf{E}$, where $c_{\text {ion }}(z)$ is the local counter-ion concentration. The solution of Stokes equation now satisfies:

$$
v(z)=v\left(L_{\text {hyd }} / 2\right)-\frac{e E}{\eta} \int_{L_{h y d} / 2}^{z} \int_{0}^{z^{\prime}} c_{i o n}\left(z^{\prime \prime}\right) \mathrm{d} z^{\prime \prime} \mathrm{d} z^{\prime}
$$

where $v\left(L_{\text {hyd }} / 2\right)$ is again determined by the boundary conditions. The ionic density profile can be determined either from molecular simulations or from an analytical theory such as PoissonBoltzmann. The predictions of the latter model, presented in appendix, will be discussed below. 


\section{Hydrodynamic boundary conditions from molecular dynamics}

In this section, we assess the validity of continuous theories by comparing analytical velocity profiles with those obtained in molecular dynamics simulations. When such a description is appropriate, this comparison further allows to determine the relevant hydrodynamic boundary conditions (non-slip or slip) and provides the parameters associated with the continuous model, such as the viscosity of the confined fluid or the slip length. We first analyze the hydrodynamic flow and determine the corresponding boundary conditions. We then use the same conditions and parameters to model the electro-osmotic flow, as a validation of these features. We finally discuss the relevance of Poisson-Boltzmann theory for the prediction of electro-osmotic flows in the present case.

\section{Hydrodynamics}

The velocity profiles in pyrophyllite and montmorillonite are given on Figure 2 for the two force fields CLAYFF and SSFF. In the central region of the pore (between $-15 \AA$ and $15 \AA$ where the fluid is far enough from the surfaces not to be disturbed ${ }^{41}$ ), the profile is parabolic, as predicted by Eq. 2. The viscosity can be deduced from the curvature, while the value of the velocity allows to characterize the boundary conditions.

When the fluid slips on the surface, the velocity does not vanish at the interface and a slip length $b$ can be defined as: ${ }^{42,43}$

$$
b=-\frac{v\left(L_{h y d} / 2\right)}{v^{\prime}\left(L_{h y d} / 2\right)}
$$

and determined from the molecular simulation velocity profile if $L_{h y d}$ is known. In this slip case, a natural choice to apply the boundary condition is to indentify $\pm L_{h y d} / 2$ with the positions $\pm z_{G D S}$ of the Gibbs Dividing Surfaces (GDS), which partition the space between a region of homogeneous fluid and a region without fluid:

$$
\int_{0}^{ \pm z_{G D S}}\left[\rho_{\text {bulk }}-\rho(z)\right] \mathrm{d} z=\int_{ \pm z_{G D S}}^{+\infty} \rho(z) \mathrm{d} z
$$




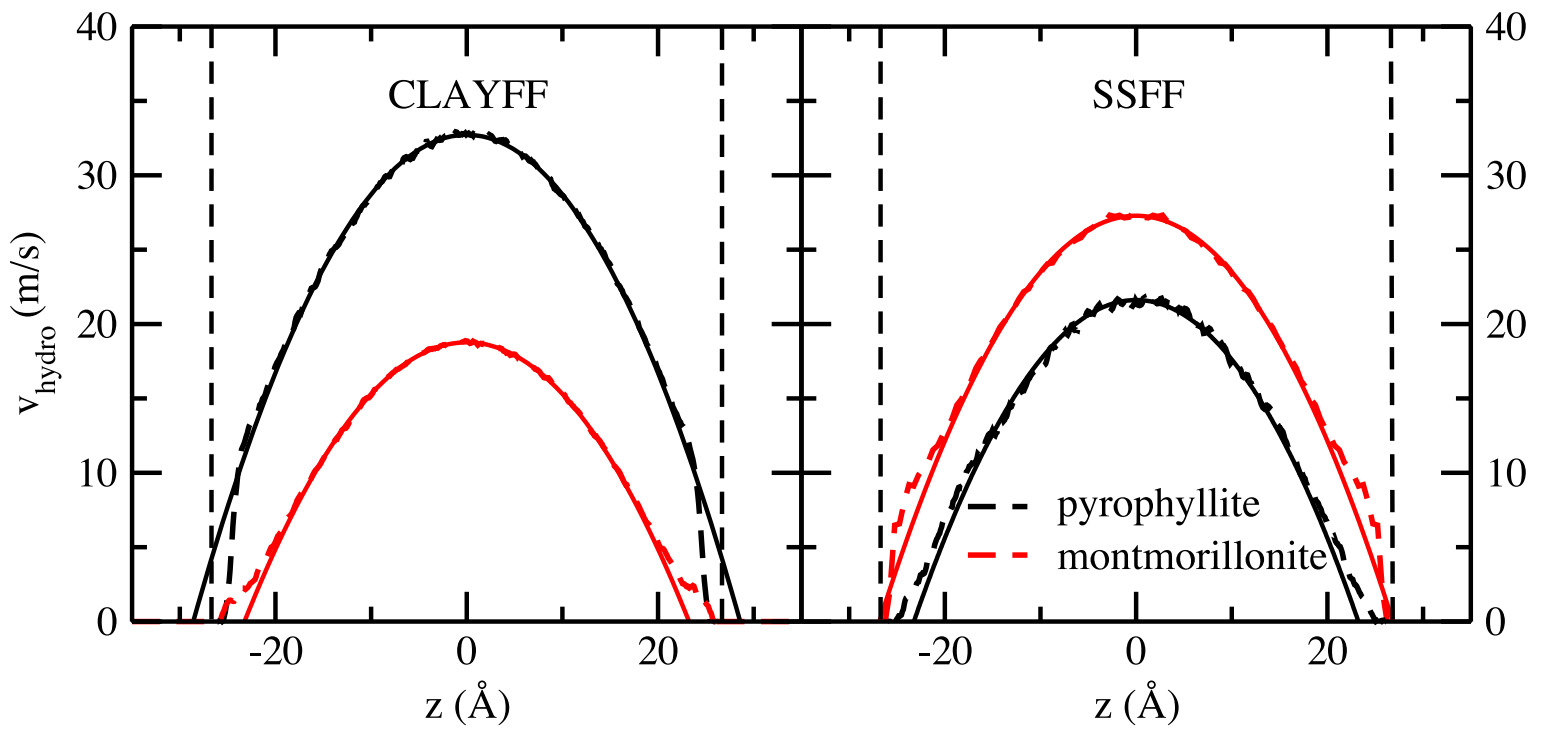

Figure 2: Hydrodynamic velocities $\left(F_{\text {hydro }}=0.75 \mathrm{cal} / \mathrm{mol} / \AA\right)$ as a function of $z$, for pyrophyllite (black) and montmorillonite (red) simulated with CLAYFF (left) and SSFF (right). The simulation results (dashed lines) are reported together with fits by parabolas (solid lines). The vertical dashed lines indicate the location of the clay surface oxygen atoms.

where $\rho_{\text {bulk }}$ and $\rho(z)$ are respectively the fluid density in the bulk, i.e. in the central region of the pore, and that at $z$. Thus $z_{G D S}$ can be calculated from the density profile of the fluid $\rho(z)$ obtained by simulation. From the profiles of Figure 2, we can conlude that this slip condition applies in the cases of pyrophyllite simulated with CLAYFF and of montmorillonite simulated with SSFF (in agreement with our previous work ${ }^{20}$ ). The values of $\eta, L_{h y d}$ and $b$ obtained from the fits are reported in Table 2 .

Table 2: Hydrodynamic parameters deduced from molecular dynamics simulations

\begin{tabular}{llcccc}
\hline & $\eta(\mathrm{cP})$ & $z_{G D S}(\AA)$ & $L_{h y d} / 2(\AA)$ & $b(\AA)$ & $d(\AA)$ \\
\hline pyrophyllite CLAYFF & $0.65 \pm 0.02$ & $24.8 \pm 0.2$ & $24.8 \pm 0.2$ & $4.1 \pm 0.2$ & \\
\hline pyrophyllite SSFF & $0.63 \pm 0.04$ & $25.8 \pm 0.2$ & $23.3 \pm 0.2$ & & $2.5 \pm 0.4$ \\
\hline montmorillonite CLAYFF & $0.72 \pm 0.02$ & $25.5 \pm 0.2$ & $23.4 \pm 0.2$ & & $2.1 \pm 0.4$ \\
\hline montmorillonite SSFF & $0.68 \pm 0.03$ & $25.5 \pm 0.2$ & $25.5 \pm 0.2$ & $1.5 \pm 0.3$ & \\
\hline
\end{tabular}

When the fluid sticks to the surfaces, a layer of thickness $d$ is immobilized at the surface. The 
positions of the shear planes are then further from the surface than the GDS:

$$
\pm \frac{L_{h y d}}{2}= \pm z_{G D S} \mp d
$$

In this stick case $v\left(L_{\text {hyd }} / 2\right)=0$, so that $L_{\text {hyd }}$ and consequently $d$ can be determined from the molecular simulation velocity profiles. As can be seen on Figure 2, the non-slip boundary condition applies in the cases of montmorillonite simulated with CLAYFF and pyrophyllite simulated with SSFF. The corresponding parameters $L_{h y d}$ and $d$ can be found in Table 2 . In both cases $d$ is of the order of one molecular diameter. The location of the GDS is also correlated with the hydrodynamic boundary conditions: As can be seen in Table 2, the slippage is more pronounced when the GDS is further from the surface oxygen atoms. This result is in agreement of the work of Wang et al., who computed $z_{G D S}$ for muscovite (hydrophilic surface) and talc (hydrophobic). ${ }^{44}$

One can first observe that the fluid viscosities deduced from the velocity profiles are all close to the bulk value for SPC/E water $\left(0.67 \mathrm{cP}^{45}\right)$. The viscosity measured for montmorillonite is only slightly larger than with pyrophyllite. Moreover, slight variations in the location of the GDS are observed depending on the considered case. The most surprising result is that the two force fields lead to opposite conclusions. In the case of CLAYFF, the fluid slips along the surfaces of pyrophyllite and sticks to those of montmorillonite, in agreement with the hydrophobic behaviour of the former which is uncharged and with the presence of hydrated counter-ion at the surface of the latter. ${ }^{46}$ Simulations with SSFF predict the opposite behaviours. In this case, the value of $b$ found with montmorillonite is in good agreement with the one reported in a smaller pore. ${ }^{20}$ Even if the values for $b$ and $d$ are small, the prediction of less slippage with pyrophyllite than with montmorillonite is unphysical. The present study thus supports the idea that CLAYFF should be preferred to SSFF when considering dynamical quantities. However, as will be shown below, the comparison between both force fields provides useful insights into the microscopic origin of the hydrodynamic behaviour of water at clay surfaces. 


\section{Electro-osmosis}

In order to check that the same boundary conditions apply in a different situation, we now analyze the electro-osmotic flows in montmorillonite. The ionic density profiles $c_{i o n}(z)$ from molecular simulation are introduced in Eq. 3, together with the slip length $b$ or stagnation length $d$ determined in the previous section. The resulting velocity profiles are compared to that obtained in NEMD simulations in Figure 3. For comparison, we also consider the solution of the same equation assuming a priori boundary conditions ( $b=0$ and $d=0$ ) or using the Poisson-Boltzmann ionic density profile (see the appendix for the determination of the latter).

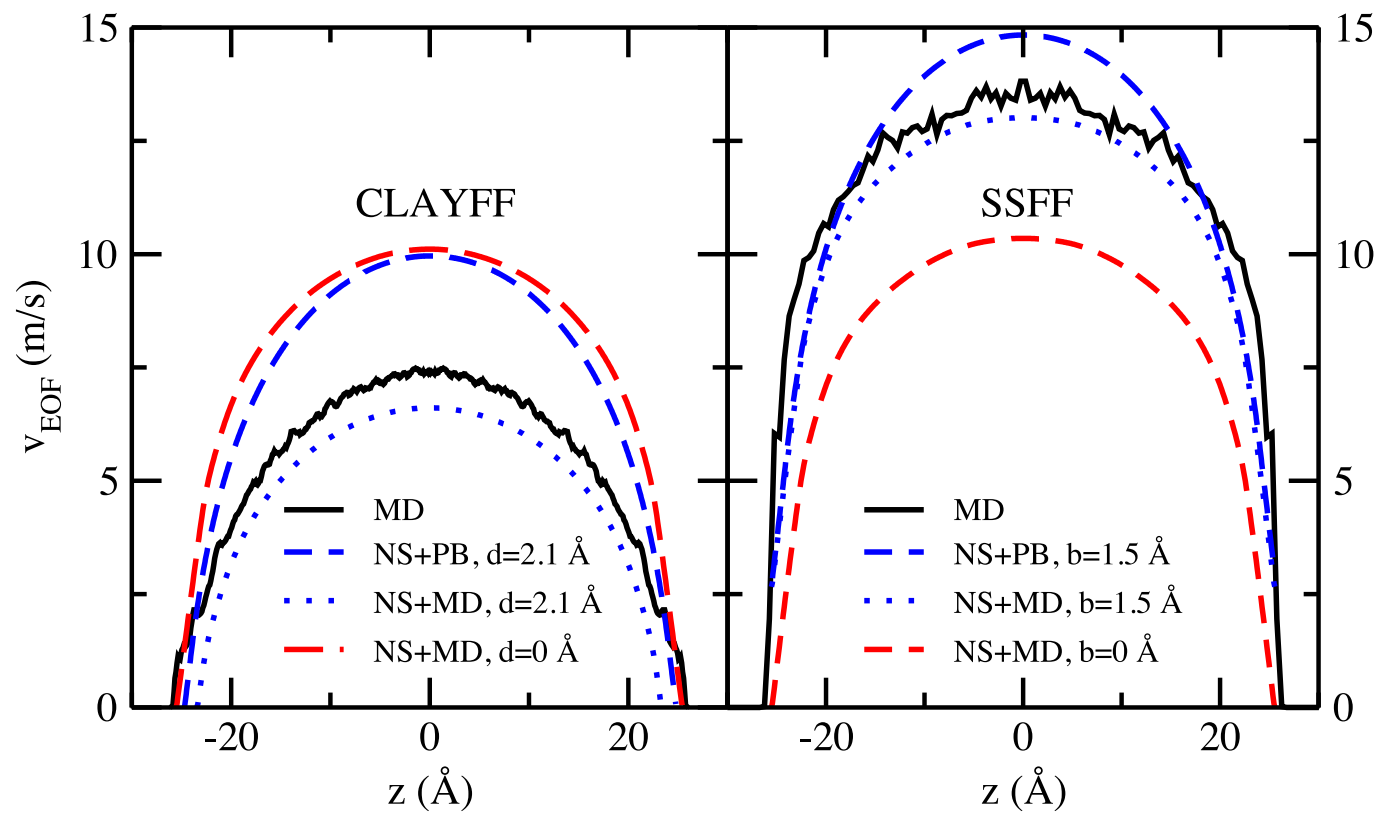

Figure 3: Electro-osmotic velocities as a function of $z$ in montmorillonite. The simulation results (solid line) are compared to the predictions of Eq. 3 for different situations: Using the ionic density profile from molecular dynamics (MD) or from Poisson-Boltzmann theory (PB), including or not the boundary conditions determined from the hydrodynamic flows (slip length $b$ or stagnation length $d$ ).

One can first notice a good agreement between the solution of Stokes equation and the MD results when the ionic density profile is taken from MD and when the boundary conditions for the hydrodynamic flow are used. This further supports the above conclusions on the slip/non-slip 
behaviour of montmorillonite as predicted by the two force fields and underlines the relevance of the corresponding parameters. Indeed, neglecting the slip or stagnation length deteriorates the prediction of continuous description. Even if these lengths are small, their influence extends far from the surface: As an example, we had shown in ${ }^{20}$ for montmorillonite simulated with SSFF that the error on the overall hydrodynamic flux is as high as $15 \%$ even for a large pore of 8 $\mathrm{nm}$. The influence of the boundary conditions is even more important for electro-osmosis because the driving force in that case is very localized near the charged surfaces. ${ }^{18,19}$ Finally, as can be seen in Figure 3, PB theory is insufficient to predict the electro-osmotic flow, even if the correct hydrodynamic boundary conditions are included, as a result of the poor description of the ionic density profile, and hence of the force acting on the fluid. Similar conclusions had been reached with model surfaces and a simple LJ fluid by Joly $a l .{ }^{47}$ or water by Huang et al. ${ }^{48}$ The discrepancy is expected to increase with surface charge density and cation valency, as in Qiao and Aluru, ${ }^{49}$ who even observed a charge reversal at a highly charged surface.

\section{Microscopic origin of the boundary conditions}

In this section, we elucidate the microscopic origin of the non-slip or slip behaviour of the clay surfaces by analyzing the change in boundary conditions $b$ and $d$ under different simulation conditions. The only difference between pyrophyllite and montmorillonite, which are expected to be hydrophobic and hydrophilic, respectively, is the presence in montmorillonite of negative charges in the middle of the clay layer, arising from the substitution of $\mathrm{Al}^{3+}$ by $\mathrm{Mg}^{2+}$, and of the counterions $\left(\mathrm{Na}^{+}\right.$in our case). One can envision two main reasons for the differences of behaviour between the two clays, which both result from the presence of this charge. (a) The hydrated cations close to the surface change the structure and dynamics of water in their hydration shell. Moreover, the presence of the cation may disturb the hydrogen bond network of water in the surface layer. (b) The net negative charge of the surface and the positive layer of counterions at the surface result in an electric field, experienced by the water molecules in between, which influences their orien- 
tation, and hence the hydrogen bond network, both between the surface water molecules and the surface and among surface molecules. ${ }^{6,41,50,51}$

Both force fields predict more hydrogen bonds with the surface in the case of montmorillonite, compared to pyrophyllite, and a similar number of such bonds for a given clay. However nonslip or slip is observed depending on the force field. Thus the number of hydrogen bonds is not sufficient to determine the hydrodynamic behaviour. In the following, we first analyze the role of the net charge and the resulting electric field by comparing montmorillonite and pyrophyllite. Then we discuss the role of the polarity of the charge distribution inside the clay layer by comparing the two force fields in the case of montmorillonite.

\section{Montmorillonite vs pyrophyllite: role of the average electric field}

The net charge of the layers and the counterions in montmorillonite generate an electric field experienced by surface water molecules. In order to investigate how this field influences the boundary conditions independently of the presence of the cations (which may modify the water structure in their solvation shell), we simulate hydrodynamic flows between pyrophyllite surfaces in the presence of an applied electric field perpendicular to the surfaces $\mathbf{E}=E \mathbf{e}_{\mathbf{z}}$. The profiles are shown on Figure 4 for both force fields for various values of the electric field $E=0.5$ and $1.5 \mathrm{~V} / \AA$, together with the profiles obtained previously in the absence of electric field. The presence of $\mathbf{E}$ induces a dissymmetry of the system which is visible on the velocity profiles. The latter can still be described by parabolas. From the curvature, we deduce the viscosity of the fluid and observe an increase with increasing $\mathbf{E}: \eta=0.80 \mathrm{cP}$ (resp. $0.72 \mathrm{cP}$ ) for $E=0.5 \mathrm{~V} / \AA$ with SSFF (resp. CLAYFF) and $\eta=0.85 \mathrm{cP}$ (resp. $0.88 \mathrm{cP}$ ) for $E=1.5 \mathrm{~V} / \AA ̊$ with SSFF (resp. CLAYFF). Indeed,

applying $\mathbf{E}$ through the whole fluid changes the nature of the fluid also in the middle of the pore, by modifying the orientation of the water molecules and hence the hydrogen bond network.

However, the most striking result is that the boundary conditions change: for CLAYFF, the fluid sticks to the left surface although it slipped in the absence of electric field; for SSFF, the fluid which stuck to the surfaces now slips on the right surface. These effects also coincide with 


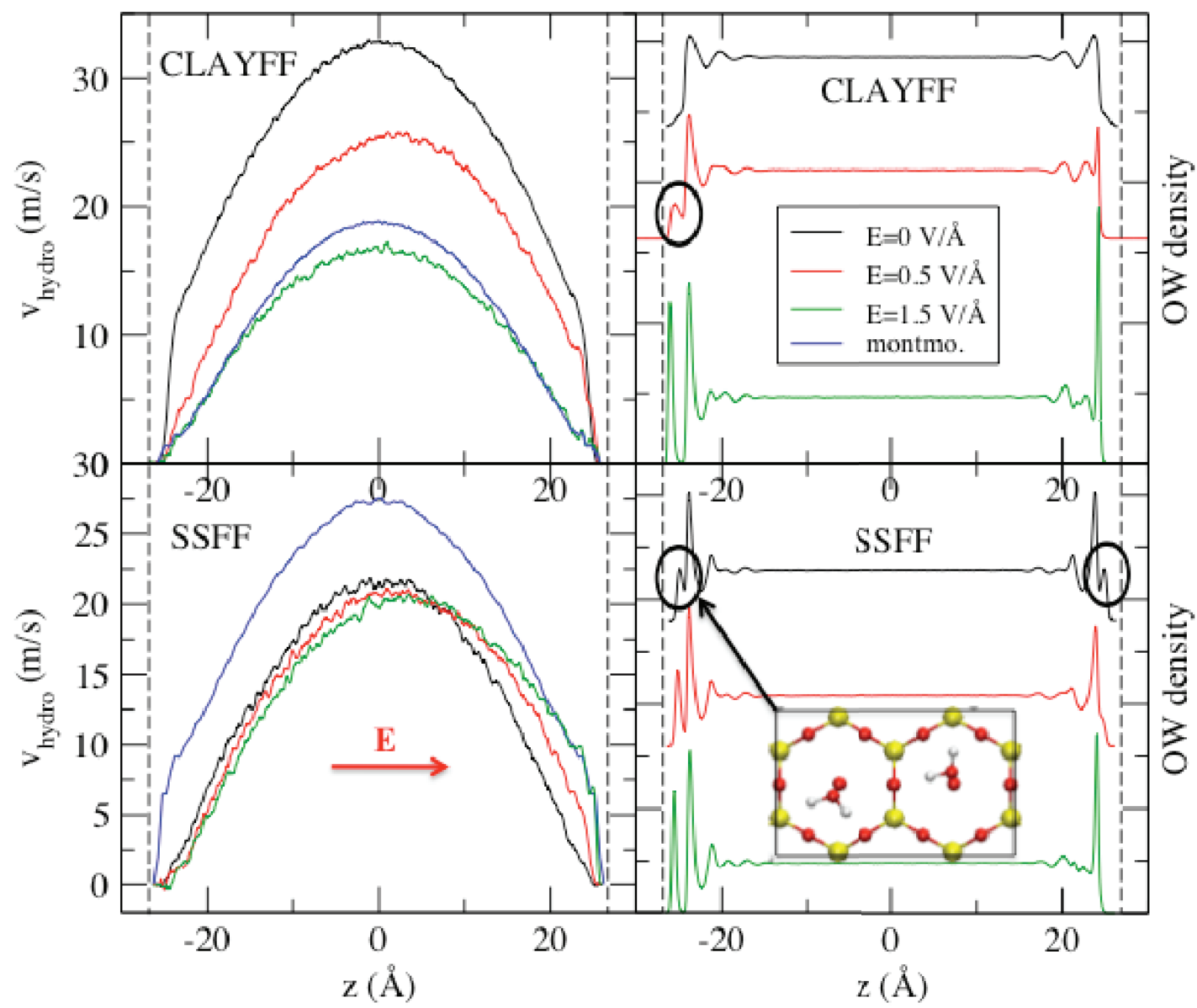

Figure 4: Hydrodynamic flow in pyrophyllite under a force $F_{\text {hydro }}=0.75 \mathrm{cal} / \mathrm{mol} / \AA$ in the presence of an applied field $\mathbf{E}$ perpendicular to the surfaces. The velocity profiles (left) and water oxygen densities (right) are reported for $E=0$ (black) 0.5 (red) and $1.5 \mathrm{~V} / \AA$ (green). The velocity profiles for montmorillonite in the absence of electric field are also indicated (blue). The vertical dashed lines indicate the location of the clay surface oxygen atoms. 
the appearance (resp. disappearance) of a small peak in the water oxygen atoms density profiles with CLAYFF (resp. SSFF), which corresponds to water molecules above hexagonal cavities, as illustrated on Figure 4. The molecules in this region are approximately horizontal with hydrogen atoms pointing slightly away from the surface. In the absence of an applied field, such molecules are present with SSFF but not with CLAYFF.

When applying $\mathbf{E}$, the water molecules tend to orientate their dipole in the direction of the field. On the left surface, this causes water molecules to turn their dipole away from the surface. In the case of CLAYFF, this new orientation seems to facilitate a closer approach to the surface and the entrance of molecules above the cavities. In the case of SSFF, this increases the occupancy of these sites. On the contrary, on the right surface, water molecules orientate with their hydrogen atoms closer to the surface than their oxygen. With SSFF, this reversal of orientation of the water molecules above hexagonal cavities causes their exit from this position.

All these observations point to a strong correlation between the presence of water molecules on these sites in the case of SSFF and the unexpected behaviour of the fluid at the surface of pyrophyllite. These molecules pinned down to preferential sites on the surface (by stronger interactions than with CLAYFF) may slow down the fluid by donating hydrogen bonds to other water molecules. This would also explains why the fluid slips more in montmorillonite when simulated with SSFF. Indeed, the first layer of water molecules is located between the negatively charged clay surfaces and the first peak of the cation density (see Figure 5). Thus these water molecules experience an electric field directed toward the surface, which corresponds to the situation at the right surface with the applied field. As a consequence, water molecules can orientate with their hydrogen atoms toward the surface and form hydrogen bonds with it, as already observed in montmorillonite. ${ }^{41}$ In addition, at this surface the electric field causes the molecules above the cavities to exit and one observes slip for montmorillonite with SSFF. This further suggests that these molecules are at least partly responsible for the non-slip boundary conditions in pyrophyllite with this force field.

The change of boundary conditions on the right surface for CLAYFF is less obvious to explain: an increase in $E$ results in a wider immobilized layer. However, when looking in details at the den- 


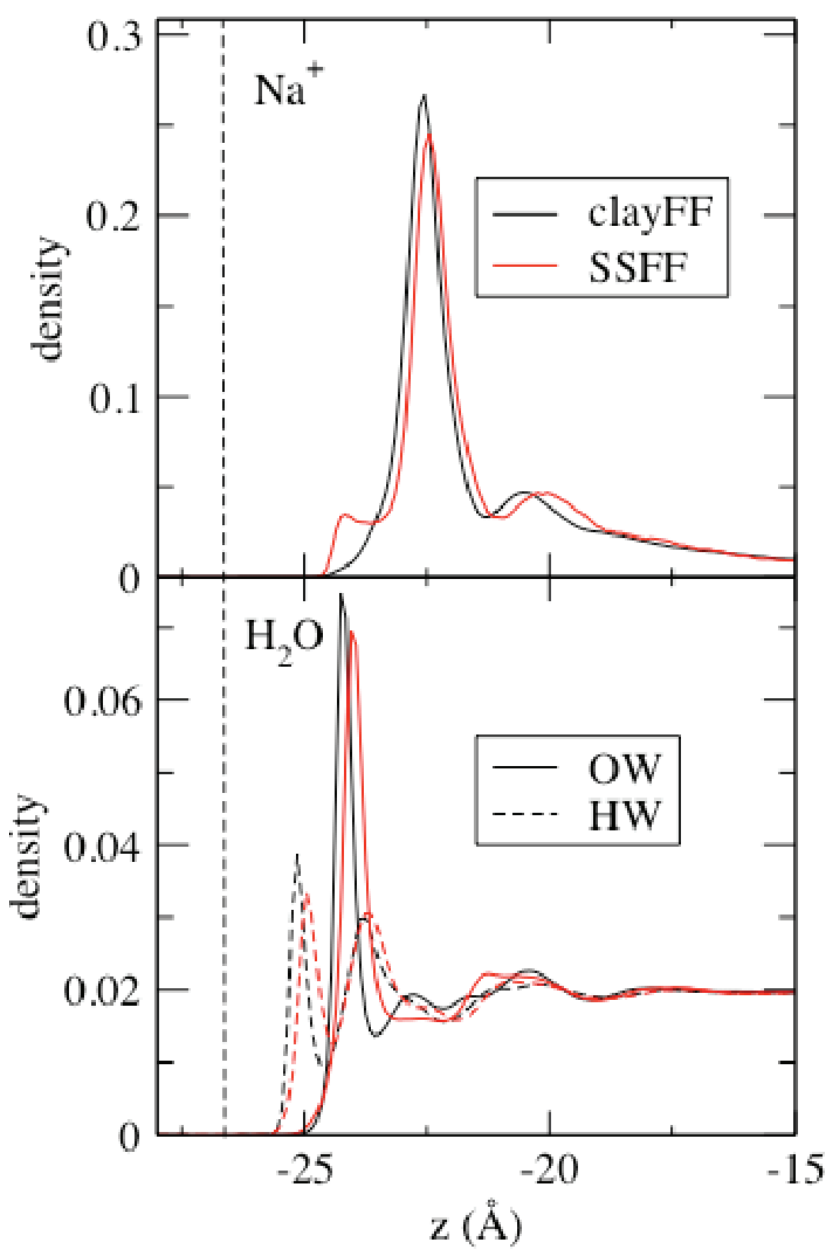

Figure 5: Density profiles in montmorillonite for the CLAYFF (black) and SSFF (red) force fields: $\mathrm{Na}^{+}$(top), water oxygen atoms (bottom, solid lines) and water hydrogen atoms (bottom, dashed lines). The vertical dashed lines indicate the location of the clay surface oxygen atoms. 
sity profiles of water oxygen atoms given on Figure 6, one can notice a strong similarity between the densities in pyrophyllite with $E=1.5 \mathrm{~V} / \AA$ and that in montmorillonite, for both force fields, at least close to the surfaces. This can be understood by considering the electric field experienced by surface water molecules in montmorillonite. Assuming first that they are located in a plane capacitor of surface charge density $\sigma= \pm 0.008071 e / \AA^{2}$ (between the negative plane inside the clay layer containing the substitutions and the positive plane containing the counter-ions), one can estimate that the field is approximately $\sigma / \varepsilon_{0} \sim 1.45 \mathrm{~V} / \AA$, which corresponds well to the above value. A more accurate estimate can be obtained by integrating Poisson equation with the cation density profiles from molecular simulations. In the considered region (corresponding to the first peak of water oxygen densities), the electrostatic fields are on average 1.47 and $1.42 \mathrm{~V} / \AA$ for CLAYFF and SSFF, respectively. Thus the molecules at the surface of montmorillonite experience an average electric field similar to those at the right surface of pyrophyllite with $E=1.5 \mathrm{~V} / \AA$, resulting in similar hydrodynamic velocity profiles.

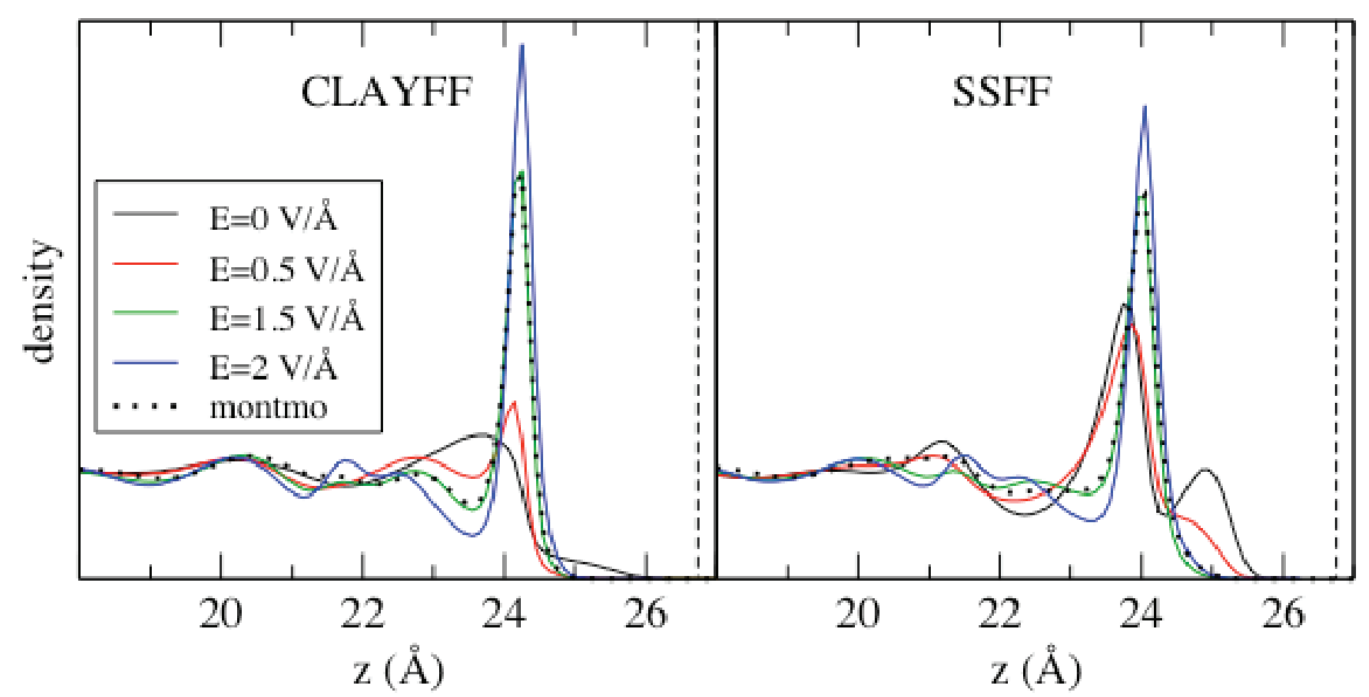

Figure 6: Density profile for water oxygen atoms in pyrophyllite with CLAYFF (left) and SSFF (right), for various values of applied field $E$ in the direction perpendicular to the surface: 0 (black), 0.5 (red), 1.5 (green) and 2 V/Å (blue). Results for montmorillonite in the absence of electric field are also indicated (dotted lines). The vertical dashed lines indicate the location of the clay surface oxygen atoms.

The main conclusion of this section is thus that the average electric field plays a key role in 
determining the hydrodynamic boundary conditions. The resulting reorientation of surface water molecules largely explains the differences observed between montmorillonite and pyrophyllite. Although the average electric field created by the charge separation seems to be a sufficient explanation without invoking the disturbance of surface water molecules in the hydration shell of cations, the role of the latter is not trivial. Indeed, their occurrence as fully hydrated ions (outer sphere complexes) in the $\mathrm{Na}^{+}$case results from the competition between solvation and attraction to the surface. For other ions $\left(e . g . \mathrm{Cs}^{+}\right)$and other surfaces (e.g. more highly charged or with substitution in the tetrahedral layer, closer to the surface), inner sphere complexes can be observed. This would in turn modify the electric field experienced by the surface water molecules, hence the hydrodynamic behaviour.

\section{Comparing force fields: role of local interactions}

While the previous analysis allows to understand the difference between pyrophyllite and montmorillonite, it remains to clarify the origin of the differences between CLAYFF and SSFF in montmorillonite. To that end, we finally compare the results obtained with both force fields in that case. Although the field experienced by the first layer of water is almost the same in both cases, the fluid sticks with CLAYFF and slips with SSFF. In fact, the comparison of the densities represented on Figure 5 reveals that the structure of the fluid is slightly different for the two force fields: The peaks are larger and closer to the surface with CLAYFF. This suggests that the fluid is more tighlty bound to the surface in that case, as a result of the differences existing between the two force fields (partial charges and Lennard-Jones parameters).

One of the reasons for a slowing down of the fluid with CLAYFF compared to SSFF can be found in differences in the strength of the hydrogen bonds formed with the surface. Indeed, the number of hydrogen bonds formed with the surface is almost the same for both force fields: 2.6 and 2.5 per unit cell for CLAYFF and SSFF, respectively. In order to roughly estimate their strength, we calculate the average force exerted on a water hydrogen atom, either bonded to another water molecule $\mathrm{O}_{W}$ or to a surface oxygen atom $\mathrm{O}_{S}$. For CLAYFF, the average forces are $4.75 \mathrm{kcal} / \mathrm{mol} / \AA$ 
when bonded to an $\mathrm{O}_{S}$ and $4.69 \mathrm{kcal} / \mathrm{mol} / \AA$ with $\mathrm{O}_{W}$, indicating that the interactions with the surface are stronger than with the surrounding water molecules. For SSFF, the average forces are $4.26 \mathrm{kcal} / \mathrm{mol} / \AA$ with $\mathrm{O}_{S}$ and $4.69 \mathrm{kcal} / \mathrm{mol} / \AA$ with $\mathrm{O}_{W}$, i.e. an opposite situation. This is likely a consequence of the fact that the charge of SPC/E oxygen $(-0.8476 e)$ is somewhat smaller than that for $\mathrm{O}_{S}$ in CLAYFF $(-1.05 e)$ and larger with SSFF $(-0.8 e)$. This explanation was already invoked in our previous work on fluoro-hectorite. ${ }^{9}$ The fact that water molecules at the surface interact more strongly with the surface than in the bulk with CLAYFF explains at least partially why the fluid is slowed down in that case. It is also illustrates from the dynamic point of view the role of the competition between adhesive (surface-water) and cohesive (water-water) interactions in determining the hydrophilic/hydrophobic behaviour of a surface, as recently analyzed on pyrophyllite, talc and fluorotalc. ${ }^{52}$

\section{Conclusion}

We investigated hydrodynamic and electro-osmotic flows in clay nanopores using NEMD simulations, to understand the role of electrostatic interactions on the hydrodynamic boundary conditions. For this purpose, we simulated charged (montmorillonite) and uncharged (pyrophyllite) clays using two commonly used force fields: SSFF and CLAYFF. For the $6 \mathrm{~nm}$ pore investigated here, the molecular velocity profile can be well described by the solution of the Stokes equation only if (a) proper boundary conditions, with a slip or stagnation length determined from molecular simulation, are taken into account and (b) the ionic density profiles from MD simulations are used in the case of electro-osmotic flow, since the Poisson-Boltzmann equation fails to reproduce the ionic profiles, hence the force acting on the fluid. While the CLAYFF force field predicts a hydrophobic pyrophyllite and hydrophilic montmorillonite as expected from experimental behaviour, the SSFF force field leads to the opposite conclusions.

The non-slip or slip boundary conditions at clay surfaces strongly depend on electrostatic interactions of surface water molecules with the surface, both on the presence/absence of a net charge 
and on the details of the charge distribution inside the clay layer. The main effect of the net charge is the resulting average electric field experienced by surface water molecules between the charged surface and the condensed layer counter-ions, which influences their orientation. The charge distribution inside the clay layer determine the polarity of the surface and hence the strength of hydrogen bonds donated by water molecules to surface oxygen atoms. The competition between hydration and attraction to the surface determines the location of counter-ions at the surface (inner or outer sphere complexes) which should in turn impact the field experienced by surface water and hence hydrodynamic boundary condition. While a systematic study is out of the scope of the present work, we are currently investigating other ions and different clays, e.g. with a larger surface charge or with a charge located in the tetrahedral layer, closer to the fluid. These conclusions should also hold for other types of surfaces and the present study provides insight into the microscopic mechanisms at play at the interface between water and charged surfaces in general, and oxides such as silica in particular. In the latter case, hydrodynamic boundary conditions will also be affected by the rugosity arising from the amourphous nature of this material. ${ }^{53}$

\section{Acknowledgement}

The authors acknowledge financial support from the Agence Nationale de la Recherche under grant ANR-09-SYSC-012 and from the Groupement National de Recherches PARIS. The authors thank Jean-François Dufrêche for useful discussions.

\section{Appendix}

In this appendix, we discuss the relevance of Poisson-Boltzmann (PB) theory for the description of ionic profiles at the surface of Na-montmorillonite in the absence of added salt. Combining Poisson equation with a Boltzmann distribution of point ions results in the PB equation:

$$
\Delta \psi=-\frac{A e}{\varepsilon_{0} \varepsilon_{r}} \exp \left(-\frac{e \psi(z)}{k_{\mathrm{B}} T}\right)
$$


where $A$ is a constant which can be determined from the electroneutrality condition. The electrostatic potential $\psi(z)$ and ion concentration $c_{i o n}(z)$ can be calculated analytically in the present case. ${ }^{54}$ For that purpose, one has to define a distance $L_{e l}$ where the boundary conditions $\psi^{\prime}\left( \pm L_{e l} / 2\right)=$ $\pm \sigma / \varepsilon_{0} \varepsilon_{r}$ apply. This distance coincides with $L$ for point ions, but otherwise differs due to ther finite size. ${ }^{18,19}$ Moreover, $L_{e l}$ and $L_{h y d}$ defined in the main text are a priori different. The solution then reads:

$$
c_{i o n}(z)=\frac{1}{2 \pi L_{\mathrm{B}}} \frac{\alpha^{2}}{\cos ^{2}(\alpha z)}
$$

where $\alpha$ is defined by $\alpha \tan \left(\alpha L_{e l} / 2\right)=-2 \pi L_{\mathrm{B}} \sigma / e$, with $L_{\mathrm{B}}=e^{2} / 4 \pi \varepsilon_{0} \varepsilon_{r} k_{\mathrm{B}} T$ the Bjerrum length. For the comparison with molecular simulations, we use $\sigma=-0.008071 e / \AA^{2}$ and the value $\varepsilon_{r}=$ 71.5, which is the dielectric constant of bulk SPC/E water. ${ }^{55}$

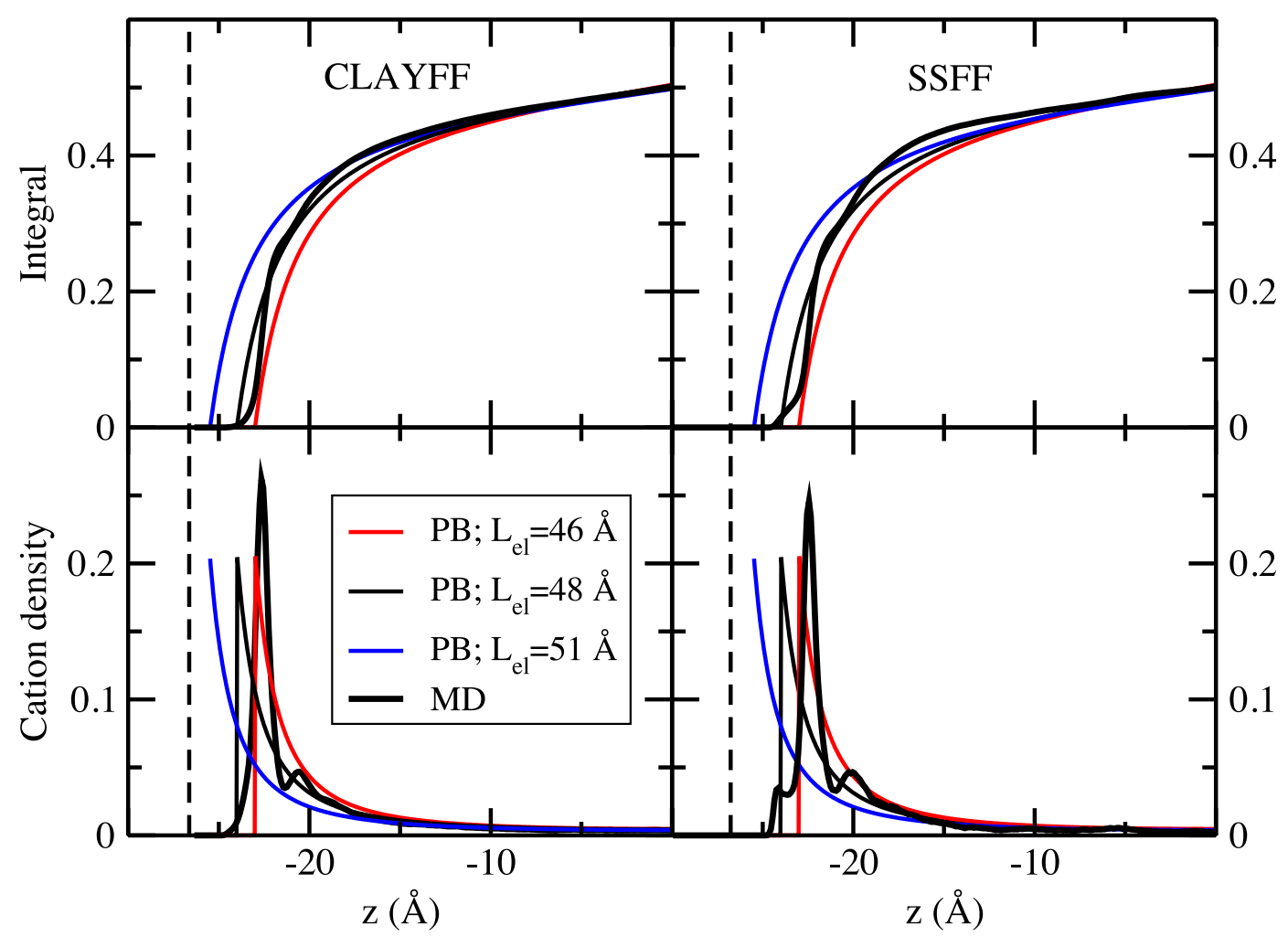

Figure 7: Cation concentration profiles and their integrals for the two force fields. The MD results (bold black line) are compared to the Poisson-Bolztmann result for $L_{e l}=46 \AA$ (red), $48 \AA$ (thin black line) and $51 \AA$ (blue line). The latter case corresponds to $L_{e l} / 2=z_{G D S}$. The vertical dashed lines indicate the location of the clay surface oxygen atoms. 
The simulated normalized cation density profiles for the two force fields are represented on Figure 7, together with calculated PB profiles for several $L_{e l}$. The profiles are very sensitive to the molecular structure of the solvent close to the surfaces. For an easier comparison with between MD and PB results, we consider the integral density, which corresponds to the fraction of cations found between $-L$ and $z$, also reported in Figure 7. One can observe that no value of $L_{e l}$ allows to reach a satisfactory agreement. Increasing $L_{e l}$ improves the description in the center of the pore but deteriorates that in the vicinity of the surfaces. As a compromise, for the calculations of electro-osmotic flows we use $L_{e l}=48 \AA$ for both force fields.

\section{References}

(1) Malikova, N.; Cadène, A.; Dubois, E.; Marry, V.; Durand-Vidal, S.; Turq, P.; Breu, J.; Longeville, S.; Zanotti, J.-M. J. Phys. Chem. C 2007, 111, 17603-17611.

(2) Malikova, N.; Cadène, A.; Marry, V.; Dubois, E.; Turq, P.; Zanotti, J.-M.; Longeville, S. Chem. Phys. 2005, 317, 226-235.

(3) Malikova, N.; Cadène, A.; Marry, V.; Dubois, E.; Turq, P. J. Phys. Chem. B 2006, 110, 32063214.

(4) M. Arab, D. Bourgeard, K. S. Smirnov, Phys. Chem. Chem. Phys. 2004, 6, 2446-2453.

(5) Michot, L.; Delville, A.; Humbert, B.; Plazanet, M.; Levitz, P. J. Phys. Chem. C 2007, 111, 9818-9831.

(6) Skipper, N. T.; Lock, P. A.; Titiloye, J. O.; Swenson, J.; Mirza, Z. A.; Howells, W. S.; Fernandez-Alonso, F. Chem. Geol. 2006, 230, 182-196.

(7) Kosakowski, G.; Churakov, S.; Thoenen, T. Clays Clay Min. 2008, 56, 190-206.

(8) Malikova, N.; Marry, V.; Dufrêche, J.-F.; Simon, C.; Turq, P.; Giffaut, E. Mol. Phys. 2004, $102,1965-1977$. 
(9) Marry, V.; Dubois, E.; Malikova, N.; Durand-Vidal, S.; Longeville, S.; Breu, J. Environ. Sci. Technol. 2011, 45, 2850-2855.

(10) Botan, A.; Rotenberg, B.; Marry, V.; Turq, P.; Noetinger, B. J. Phys. Chem. C 2010, 114, $14962-14969$.

(11) Lauga, E.; Brenner, M.; Stone, H. In Handbook of Experimental Fluid Dynamics; Foss, J., Tropea, C., Yarin, A., Eds.; Springer, 2005; Chapter Microfluidics: The No-Slip Boundary Condition.

(12) Cao, B.-Y.; Sun, J.; Chen, M.; Guo, Z.-Y. Int. J. Mol. Sci. 2009, 10, 4638-4706.

(13) Huang, D. M.; Cottin-Bizonne, C.; Ybert, C.; Bocquet, L. Langmuir 2008, 24, 1442-1450.

(14) Cho, J.-H. J.; Law, B. M.; Rieutord, F. Phys. Rev. Lett. 2004, 92, 166102.

(15) Stein, D.; Kruithof, M.; Dekker, C. Phys. Rev. Lett. 2004, 93, 035901.

(16) Dukhin, A.; Dukhin, S.; Goetz, P. Langmuir 2005, 21, 9990-9997.

(17) Joseph, S.; Aluru, N. R. Langmuir 2006, 22, 9041-9051.

(18) Marry, V.; Dufrêche, J.-F.; Jardat, M.; P.Turq, Mol. Phys. 2003, 101, 3111-3119.

(19) Dufrêche, J.-F.; Marry, V.; Maliková, N.; P.Turq, J. Mol. Liq. 2005, 118, 145-153.

(20) Boțan, A.; Rotenberg, B.; Marry, V.; Turq, P.; Noetinger, B. J. Phys. Chem. C 2011, 115, $16109-16115$.

(21) Skipper, N.; Refson, K.; McConnell, J. Clay Min. 1989, 24, 411-425.

(22) Delville, A. Langmuir 1992, 8, 1796-1805.

(23) Teppen, B.; Rasmussen, K.; Bertsch, P.; Miller, D.; Schafer, L. J. Phys. Chem. B 1997, 101, $1579-1587$. 
(24) Bougeard, D.; Smirnov, K.; Geidel, E. J. Phys. Chem. B 2000, 104, 9210-9217.

(25) Smith, D. Langmuir 1998, 14, 5959-5967.

(26) Sainz-Diaz, C.; Hernandez-Laguna, A.; Dove, M. Phys. Chem. Min. 2001, 28, 130-141.

(27) Cygan, R.; Liang, J.-J.; Kalinichev, A. J. Phys. Chem. B 2004, 108, 1255-1266.

(28) Heinz, H.; Koerner, H.; Anderson, K. L.; Vaia, R. A.; Farmer, B. Chem. Mater. 2005, 17, 5658-5669.

(29) Pitman, M. C.; van Duin, A. C. T. J. Am. Chem. Soc. 2012, 134, 3042-3053.

(30) Frenkel, D.; Smit, B. Understanding molecular simulation: from algorithms to applications, 2d edition; Academic Press, 2002.

(31) Skipper, N.; Chang, F.-R.; Sposito, G. Clays Clay Miner. 1995, 43, 285-293.

(32) Rotenberg, B.; Morel, J.-P.; Marry, V.; Turq, P.; Morel-Desrosiers, N. Geochim. Cosmochim. Acta 2009, 73, 4034-4044.

(33) Tambach, T.; Hensen, E.; Smit, B. J. Phys. Chem. B 2004, 108, 7586-7596.

(34) Smith, D.; Wang, Y.; Chaturvedi, A.; Whitley, H. J. Phys. Chem. B 2006, 110, 20046-20054.

(35) Wang, J.; Kalinichev, A.; Kirkpatrick, R.; Cygan, R. J. Phys. Chem. B 2005, 109, 1589315905.

(36) Bourg, I.; Sposito, G. Environ. Sci. Technol. 2010, 44, 2085-2091.

(37) Meleshyn, A. J. Phys.l Chem. C 2009, 113, 12946-12949.

(38) Berendsen, H.; Grigera, J.; Straatsma, T. J. Phys. Chem. 1987, 91, 6269-6271.

(39) Smith, D. E.; Dang, L. X. J. Chem. Phys. 1994, 100, 3757-3766.

(40) Plimpton, S. J. Comp. Phys. 1995, 117, 1-19, http://lammps.sandia.gov. 
(41) Marry, V.; Rotenberg, B.; Turq, P. Phys. Chem. Chem. Phys. 2008, 10, 4802-4813.

(42) Bocquet, L.; Barrat, J.-L. Phys. Rev. E 1994, 49, 3079-3092.

(43) Bocquet, L.; Charlaix, E. Chem. Soc. Rev. 2010, 39, 1073-1095.

(44) Wang, J.; Kalinichev, A. G.; Kirkpatrick, R. J. J. Phys.l Chem. C 2009, 113, 11077-11085.

(45) Tazi, S.; Boţan, A.; Salanne, M.; Marry, V.; Turq, P.; Rotenberg, B. J. Phys.: Condens. Matter 2012, 24, 284117.

(46) Chesworth, W. Encyclopedia of Soil Science; Springer, 2008.

(47) Joly, L.; Ybert, C.; Trizac, E.; Bocquet, L. J. Chem. Phys. 2006, 125, 204716.

(48) Huang, D. M.; Cottin-Bizonne, C.; Ybert, C.; Bocquet, L. Phys. Rev. Lett. 2007, 98, 177801.

(49) Qiao, R.; Aluru, N. Phys. Rev. Lett. 2004, 92, 198301.

(50) Wang, J.; Kalinichev, A.; Kirkpatrick, R. Geochim. Cosmochim. Acta 2006, 70, 562-582.

(51) Tambach, T.; Bolhuis, P.; Hensen, E.; Smit, B. Langmuir 2006, 22, 1223-1234.

(52) Rotenberg, B.; Patel, A. J.; Chandler, D. J. Am. Chem. Soc. 2011, 133, 20521-20527.

(53) Siboulet, B.; Coasne, B.; Dufrêche, J.-F.; Turq, P. J. Phys. Chem. B 2011, 115, 7881-7886.

(54) Engström, S.; H.Wennerström, J. Phys. Chem. 1978, 82, 2711-2714.

(55) Gereben, O.; Pusztai, L. Chem. Phys. Lett. 2011, 507, 80-83. 


\section{Page 27 of 27}

\section{Graphical TOC Entry}

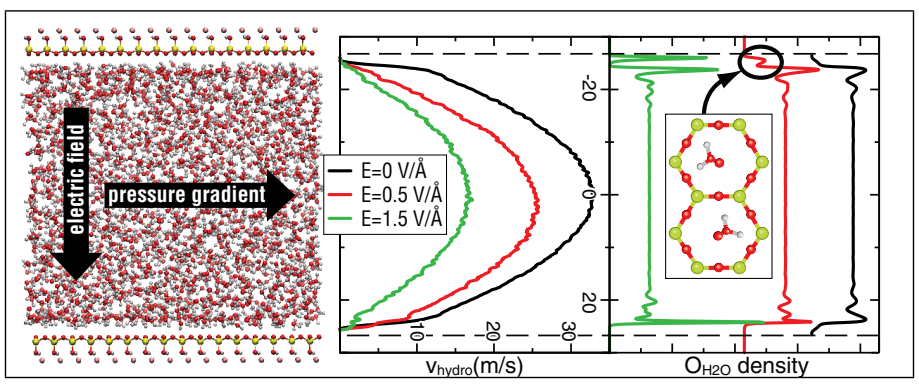

16

17

18

19

20

21

22

23

24

25

26

27

28

29

30

31

32

33

34

35

36

37

38

39

40

41

42

43

44

45

46

47

48

49

50

51

52

53

54

55

56

57

58

59

60 